Review

\title{
The Newborn Screening Quality Assurance Program at the Centers for Disease Control and Prevention: Thirty-Five Year Experience Assuring Newborn Screening Laboratory Quality
}

\author{
Víctor R. De Jesús *, Joanne V. Mei ${ }^{\dagger}$, Suzanne K. Cordovado ${ }^{\dagger}$ and Carla D. Cuthbert ${ }^{\dagger}$
}

Newborn Screening and Molecular Biology Branch, Division of Laboratory Sciences, National Center for Environmental Health, US Centers for Disease Control and Prevention, 4770 Buford Highway, NE, Mail Stop F-19, Atlanta, GA 30341, USA; E-Mails: jvm0@cdc.gov (J.V.M.); snc4@cdc.gov (S.K.C.); ijz6@cdc.gov (C.D.C.)

$\dagger$ These authors contributed equally to this work.

* Author to whom correspondence should be addressed; E-Mail: vdejesus @cdc.gov;

Tel.: +770-488-7963; Fax: +770-488-7459.

Academic Editor: Ralph Fingerhut

Received: 25 February 2015/Accepted: 27 March 2015 / Published: 17 April 2015

\begin{abstract}
Newborn screening is the largest genetic testing effort in the United States and is considered one of the ten great public health achievements during the first 10 years of the 21st century. For over 35 years, the Newborn Screening Quality Assurance Program (NSQAP) at the US Centers for Disease Control and Prevention has helped NBS laboratories ensure that their testing does not delay diagnosis, minimizes false-positive reports, and sustains high-quality testing performance. It is a multi-component program that provides comprehensive quality assurance services for dried blood spot testing. The NSQAP, the Biochemical Mass Spectrometry Laboratory (BMSL), the Molecular Quality Improvement Program (MQIP) and the Newborn Screening Translation Research Initiative (NSTRI), aid screening laboratories achieve technical proficiency and maintain confidence in their performance while processing large volumes of specimens daily. The accuracy of screening tests could be the difference between life and death for many babies; in other instances, identifying newborns with a disorder means that they can be treated and thus avoid life-long disability or severe cognitive impairment. Thousands of newborns and their families have benefited from reliable and accurate testing that has been accomplished by a network of screening laboratories and the NSQAP, BMSL, MQIP and NSTRI.
\end{abstract}


Keywords: newborn screening; quality assurance; dried blood spots; Newborn Screening Quality Assurance Program

\section{Introduction}

Newborn screening (NBS) is the largest genetic testing effort in the United States and is considered one of the ten great public health achievements during the first 10 years of the 21 st century [1]. Screening tests are designed to detect asymptomatic newborns at risk for a disease from those who are not at risk. Effective screening of newborns, combined with follow-up diagnostic confirmatory testing and treatment, helps prevent morbidity and mortality. In the United States, public health laboratories or their associated laboratories routinely screen dried blood spot (DBS) specimens for inborn errors of metabolism and other disorders that require medical intervention [2]. The Centers for Disease Control and Prevention's Newborn Screening Quality Assurance Program (NSQAP) helps NBS laboratories ensure that testing accurately detects these disorders, does not delay diagnosis, minimizes false-positive reports, and sustains high-quality testing performance [3]. For over 35 years, NSQAP has performed this essential public health service, ensuring the quality and accuracy of screening tests for more than 4 million babies born each year in the United States.

All NBS laboratories in the United States must meet strict quality assurance (QA) criteria in order to perform testing on human specimens through the Clinical Laboratory Improvement Amendments (CLIA) [4]. They must participate in proficiency testing (PT) programs designed to evaluate the quality of laboratory performance on a periodic basis using specimens in the dried blood matrix. Proficiency testing examines the analytical performance of individual laboratories for specific assays, and is used to monitor laboratories' continuing performance at one point in time. All PT challenges are designed to mimic actual newborn specimens, and thus should be assayed following the individual laboratories' standard operating procedures.

The NSQAP provides comprehensive QA services that include PT and quality control (QC) materials for DBS testing. Thus, NSQAP enables laboratories to meet the QA requirement for verifying test accuracy and provides technical guidance to participating laboratories to help assure that no screen-positive cases are misclassified during routine screening activities.

Participation in NSQAP allows laboratories to gain testing confidence through an external QA program for comparative analysis of peer performance within and among methods [2]. All PT and QC materials simulate, as closely as possible, specimens that are appropriate for a variety of assay systems. These DBS materials are certified for homogeneity, accuracy, stability, and suitability for assays from different commercial sources. Moreover, NSQAP provides training in the preparation of DBS materials and some test methods. The program staff compiles and distributes summary data reports each year for the comparative assessment of methods used by participants.

This review presents the NSQAP's expansion timeline (Section 1.1) and current program statistics (Section 1.2). In Sections 2 and 3 we present a detailed description of NSQAP's PT and QC programs, highlighting our practices in support of NBS laboratory quality. Section 4 details our program's expansion since 2009, where two new laboratories were created to enhance programmatic support to 
newborn screening laboratories. In Section 5 we present a preview of our program's future activities in response to current NBS laboratory needs.

\subsection{Newborn Screening Quality Assurance Program Timeline}

The NSQAP began providing services to newborn screening laboratories in 1978, when the first thyroxine and thyroid-stimulating hormone QC materials in DBS were distributed (Figure 1). The first PT surveys for phenylketonuria (PKU) and Congenital Hypothyroidism were conducted in 1980. That same year, the NSQAP Filter Paper Evaluation Project was established after the development of a DBS-based quantitative radio-isotopic test to measure and monitor the performance characteristics of filter paper used to collect newborn screening specimens under a standardized protocol. This work resulted in the development of the laboratory standard, Clinical and Laboratory Standards Institute NBS01-A6 Approved Standard, that addresses issues associated with specimen collection, the filter paper collection device, and the transfer of blood onto filter paper [5]. The first distribution of QC materials for phenylalanine occurred in 1983. In 1988, NSQAP began distributing HIV-1 QC and PT materials for HIV antibodies in DBS as part of the seroprevalence survey among childbearing women [6]. Galactose QC materials were initially offered in 1988, followed by the first galactosemia PT survey in 1989.

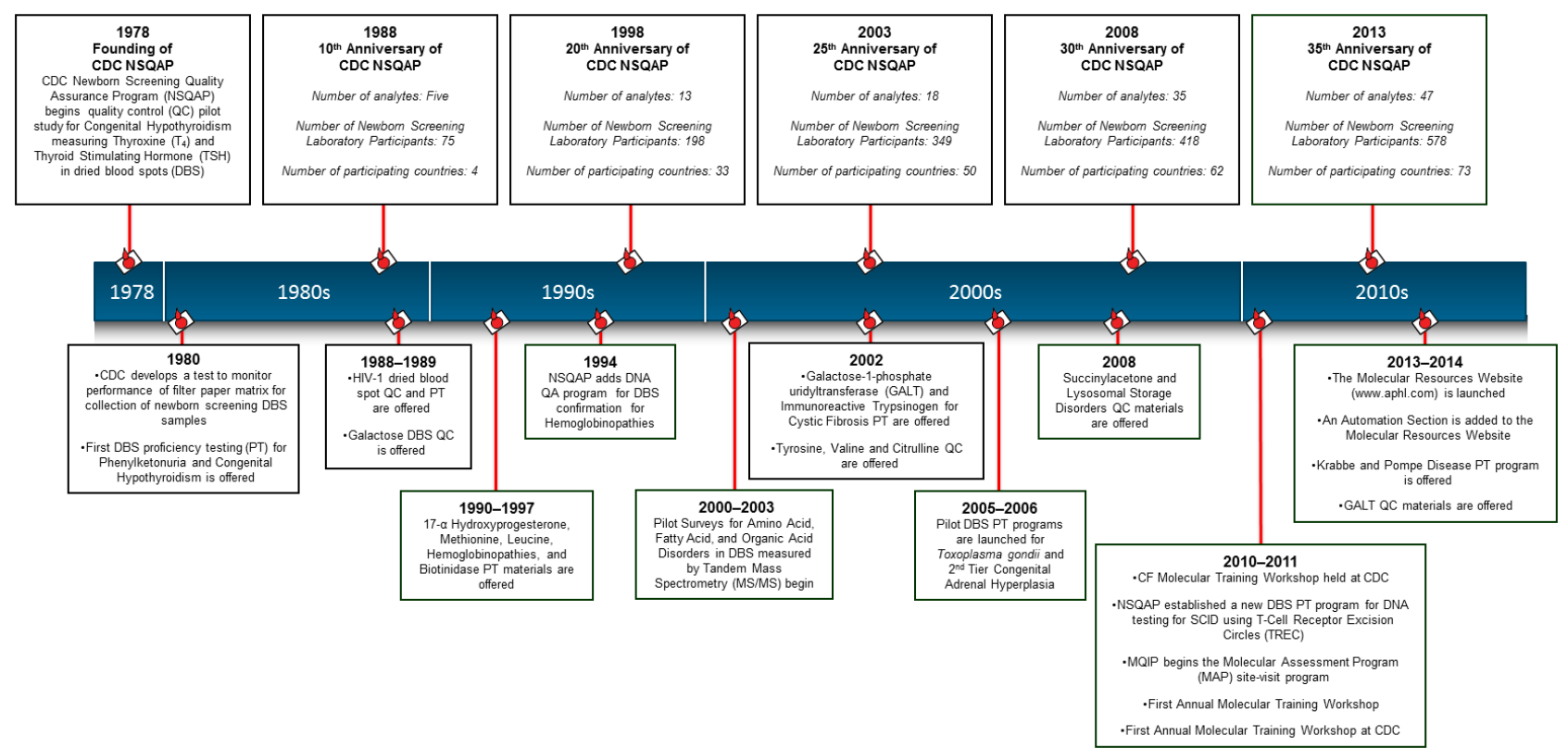

Figure 1. Newborn Screening Quality Assurance Program Timeline.

The first congenital adrenal hyperplasia (CAH) PT survey using 17- $\alpha$-hydroxyprogesterone was distributed to NSQAP participants in 1990, followed by distribution of CAH QC materials in 1991. Additionally, in 1991, proficiency testing for sickle cell disease and other hemoglobinopathies were added to the program. In 1994, NSQAP established the QA program for DNA confirmatory methods using DBS specimens for hemoglobins A, S, C, E and D. After a pilot PT survey, methionine was added in 1995 to both the growing amino acids PT panel and to the amino acid DBS QC materials. Molecular 
methods for detecting the cystic fibrosis (CF) mutation, F508del (p.Phe508del) in DBS were established in the NSQAP laboratory in 1997, along with the first biotinidase deficiency PT survey.

The introduction of tandem mass spectrometry (MS/MS)-based methods for the detection of phenylalanine in DBS [7] revolutionized the practice of newborn screening for amino acid, fatty acid oxidation, and organic acid metabolic disorders. In 2001, NSQAP launched a pilot PT survey for laboratories testing DBS by MS/MS for the detection of amino acid, fatty acid oxidation, and organic acid disorders. The program began distributing the galactose-1-phosphate uridyltransferase (GALT) PT survey, as well as panels of DBS specimens for immunoreactive trypsinogen (IRT) in 2002. In 2004, NSQAP added a PT program for DBS that mimicked CF newborn specimens with elevated IRT levels and the F508del (p.Phe508del) mutation. The program's expansion continued in 2005, when a pilot PT survey for Toxoplasma gondii antibodies was launched for laboratories performing Toxoplasmosis screening [8]. In 2006, NSQAP launched the CAH PT pilot survey to support laboratories evaluating a second tier MS/MS-based CAH assay [9], which involved the detection of other steroid markers and the establishment of a clinical algorithm to identify CAH-affected newborns. In 2007, NSQAP expanded its CF PT surveys to include separate DBS panels to test for CF mutations using molecular methods. IRT became an independent PT program and was not included in the CF Mutation Detection surveys. The expanded CF Mutation Detection survey enhanced the NSQAP's expertise in molecular biology-based technology while establishing a solid foundation to assist participating laboratories with their DNA-based screening assays.

In 2008, a PT survey for succinylacetone (a specific marker for Tyrosinemia type 1) was launched. In addition, in 2008, QC materials for lysosomal storage disorders (LSD) screening using DBS were offered to NBS laboratories, in collaboration with the Newborn Screening Translation Research Initiative (NSTRI) at CDC [10]. In 2011, NSQAP, in collaboration with NSTRI, began a PT program for T-cell receptor excision circle (TREC) analysis in DBS to detect Severe Combined Immunodeficiency (SCID). NSTRI initially worked with the Wisconsin and Massachusetts newborn screening programs to develop methods and DBS reference materials for the detection of TREC. Materials were prepared from human blood, including cord blood from unaffected individuals and modified adult blood depleted of mononuclear cells or leukocytes. Through the Model Performance Evaluation Survey (MPES) operated by NSTRI, the DBS reference materials were offered to all US and international laboratories that conducted routine NBS for SCID. Over one dozen US and international NBS laboratories evaluated the reference materials in seven collaborative surveys. Using the cumulative experience of the laboratories that tested the DBS TREC reference materials through MPES, NSQAP initiated the TREC PT survey for US laboratories-only with seven participants.

To further enhance NSQAP's molecular expertise and capacity, the Molecular Quality Improvement Program (MQIP) was created in 2011, allowing expanded molecular characterization including CFTR gene sequencing and large deletion analysis of the CF Mutation Detection PT materials (Section 4.1). In addition, in 2011, MQIP, in collaboration with the Association of Public Health Laboratories (APHL), launched a laboratory site visit program to assess the molecular components of the newborn screening laboratory. In addition, the Molecular Resources website, a joint effort of MQIP and APHL, was created in 2012, to provide screening specific molecular resources to newborn screening laboratories.

The Biochemical Mass Spectrometry Laboratory (BMSL) was created in 2011 (Section 4.2). Its mission is to work with public health laboratories, academic institutions and clinical laboratories to 
develop new MS-based assays to detect and monitor metabolic disorders, and enhance newborn screening laboratory performance through innovative approaches for biochemical marker detection.

In 2013 a pilot PT survey for two LSDs, Krabbe and Pompe disease, was started by NSTRI and NSQAP for US domestic laboratories. NSTRI prepared DBS specimens from human blood, including cord blood from unaffected individuals and leuko-depleted adult blood restored with lymphoblast cells derived from patients with either Krabbe or Pompe. The LSD PT program currently has seven participants.

The BMSL and NSQAP collaborated with the Genetic Disease Laboratory Branch of the California Department of Public Health to develop DBS QC materials for monitoring the performance of GALT screening tests [11]. The collaboration led to the launch of a GALT QC program in 2014. In addition, in 2014 the TREC PT program was expanded to all newborn screening laboratories worldwide and enrollment grew to thirty-eight laboratories.

\subsection{NSQAP Biomarker Coverage, Participant Enrollments and Program Statistics}

The NSQAP offers DBS QA materials for 26 of 31 primary (core) disorders (two disorders are screened by point-of-care technologies), and 24 of 26 secondary disorders in the US Department of Health and Human Services Recommended Uniform Screening Panel (RUSP) [12] (Tables 1 and 2). Additionally, NSQAP offers DBS QA materials for X-linked adrenoleukodystrophy (X-ALD), HIV and Toxoplasma gondii antibodies, and six lysosomal storage disorders. Moreover, DBS QA materials are offered for selected second-tier tests. These include CAH by MS/MS, CF mutation testing by DNA-based technology including 71 CFTR mutations covering all of the 23 CFTR mutations recommended for screening by the American College of Medical Genetics (ACMG), branched-chain amino acids for MSUD by MS/MS, and second-tier propionic acidemia/methylmalonic acidemia. NSQAP serves 612 laboratories in 71 countries (as of December 2014). Annually, NSQAP produces approximately one million DBS to meet the QA and PT needs of its participating newborn screening laboratories worldwide. Enrollment in the NSQAP program is open to laboratories actively screening newborn populations using the DBS matrix. A Request for Participation Form may be downloaded from NSQAP's website (http://www.cdc.gov/labstandards/nsqap.html).

Table 1. US Recommended Uniform Screening Panel Core Conditions.

\begin{tabular}{ccc}
\hline Core Condition & ACMG Code & $\begin{array}{c}\text { NSQAP QA } \\
\text { Materials (Y/N) }\end{array}$ \\
\hline Propionic acidemia & PROP & Y \\
Methylmalonic acidemia (methylmalonyl-CoA mutase) & MUT & Y \\
Methylmalonic acidemia (cobalamin disorders) & Cbl A,B & Y \\
Isovaleric acidemia & IVA & Y \\
3-Methylcrotonyl-CoA carboxylase deficiency & 3-MCC & Y \\
3-Hydroxy-3-methyglutaric aciduria & HMG & Y \\
Holocarboxylase synthase deficiency & MCD & Y \\
$\beta-$ Ketothiolase deficiency & $\beta K T$ & Y \\
Glutaric acidemia type I & GA1 & Y \\
Carnitine uptake defect/carnitine transport defect & CUD & Y \\
Medium-chain acyl-CoA dehydrogenase deficiency & MCAD & Y \\
Very long-chain acyl-CoA dehydrogenase deficiency & VLCAD & Y \\
\hline
\end{tabular}


Table 1. Cont.

\begin{tabular}{ccc}
\hline Core Condition & ACMG Code & $\begin{array}{c}\text { NSQAP QA } \\
\text { Materials (Y/N) }\end{array}$ \\
\hline Long-chain L-3 hydroxyacyl-CoA & LCHAD & $\mathrm{Y}$ \\
dehydrogenase deficiency & TFP & $\mathrm{Y}$ \\
Trifunctional protein deficiency & ASA & $\mathrm{Y}$ \\
Argininosuccinic aciduria & $\mathrm{CIT}$ & $\mathrm{Y}$ \\
Citrullinemia type I & MSUD & $\mathrm{Y}$ \\
Maple syrup urine disease & $\mathrm{HCY}$ & $\mathrm{Y}$ \\
Homocystinuria & PKU & $\mathrm{Y}$ \\
Classic phenylketonuria & $\mathrm{TYR} \mathrm{I}$ & $\mathrm{Y}$ \\
Tyrosinemia type I & $\mathrm{CH}$ & $\mathrm{Y}$ \\
Primary congenital hypothyroidism & $\mathrm{CAH}$ & $\mathrm{Y}$ \\
Congenital adrenal hyperplasia & $\mathrm{BIOT}$ & $\mathrm{Y}$ \\
Biotinidase deficiency & $\mathrm{CF}$ & $\mathrm{Y}$ \\
Cystic fibrosis & $\mathrm{GALT}$ & $\mathrm{Y}$ \\
Classic galactosemia & $\mathrm{SCID}$ & $\mathrm{Y}$ \\
Severe combined immunodeficiencies & $\mathrm{Hb} \mathrm{SS}$ & $\mathrm{N}$ \\
S,S Disease (Sickle cell anemia) & $\mathrm{Hb} \mathrm{S/ \beta} \mathrm{Th}$ & $\mathrm{N}$ \\
S, beta-Thalassemia & $\mathrm{Hb} \mathrm{S/C}$ & $\mathrm{N}$ \\
S,C Disease & $\mathrm{CCHD}$ & $\mathrm{N} / \mathrm{A}$ \\
Critical congenital heart disease & $\mathrm{HEAR}$ & $\mathrm{N} / \mathrm{A}$ \\
Hearing loss & & \\
\hline
\end{tabular}

Table 2. US Recommended Uniform Screening Panel Secondary Conditions.

\begin{tabular}{ccc}
\hline Secondary Condition & ACMG Code & $\begin{array}{c}\text { NSQAP QA } \\
\text { Materials (Y/N) }\end{array}$ \\
\hline Methylmalonic acidemia with homocystinuria & Cbl C,D & Y \\
Malonic acidemia & MAL & Y \\
Isobutyrylglycinuria & IBG & Y \\
2-Methylbutyrylglycinuria & 2MBG & Y \\
3-Methylglutaconic aciduria & 3MGA & Y \\
2-Methyl-3-hydroxybutyric aciduria & 2M3HBA & Y \\
Short-chain acyl-CoA dehydrogenase deficiency & SCAD & Y \\
Medium/short-chain L-3-hydroxyacyl-CoA dehydrogenase & M/SCHAD & Y \\
deficiency & GA2 & Y \\
Glutaric acidemia type II & MCAT & Y \\
Medium-chain ketoacyl-CoA thiolase deficiency & DE RED & Y \\
2,4 Dienoyl-CoA reductase deficiency & CPT IA & Y \\
Carnitine palmitoyltransferase type I deficiency & CPT II & Y \\
Carnitine palmitoyltransferase type II deficiency & CACT & Y \\
Carnitine acylcarnitine translocase deficiency & ARG & Y \\
Argininemia & CIT II & Y \\
Citrullinemia type II & MET & Y \\
Hypermethioninemia & H-PHE & Y \\
Benign hyperphenylalaninemia & &
\end{tabular}


Table 2. Cont.

\begin{tabular}{ccc}
\hline Secondary Condition & ACMG Code & $\begin{array}{c}\text { NSQAP QA } \\
\text { Materials (Y/N) }\end{array}$ \\
\hline Biopterin defect in cofactor biosynthesis & BIOPT (BS) & Y \\
Biopterin defect in cofactor regeneration & BIOPT (REG) & Y \\
Tyrosinemia type II & TYR II & Y \\
Tyrosinemia type III & TYR III & Y \\
Various other hemoglobinopathies & Var Hb & Y \\
Galactoepimerase deficiency & GALE & N \\
Galactokinase deficiency & GALK & N \\
T-cell related lymphocyte deficiencies & - & Y \\
\hline
\end{tabular}

\section{NSQAP Proficiency Testing Program}

The NSQAP provides NBS laboratories with quarterly panels of five blind-coded DBS specimens in all PT programs (Table 3). Data is returned to the program through either a web-based, secured reporting system, or by email. Reports of individual laboratory performance and summary data by analyte and method are available within one week of the closure of the internet data-reporting system. For PT challenges, laboratories report the cutoff value for each analyte tested. While there is variability in cutoff values among laboratories, participants are evaluated by their choice of whether a specimen's results are in-range or out-of-range based on their laboratory cutoff. The reported analytical values are also an important component of the overall grading algorithm because specimen assessments are based on the expected analytical values [3].

Table 3. NSQAP Proficiency Testing (PT) Programs as of December 2014.

\begin{tabular}{|c|c|}
\hline PT Program (Number of Participants) & Analytes Included in Each Program \\
\hline Amino Acids (444) & $\begin{array}{l}\text { Arginine, Leucine, Phenylalanine, Tyrosine, } \\
\text { Valine, Citrulline, Succinylacetone, Methionine }\end{array}$ \\
\hline Acylcarnitines (315) & $\begin{array}{c}\mathrm{C} 0(\mathrm{~L}), \mathrm{C} 3, \mathrm{C} 3 \mathrm{DC}, \mathrm{C} 4, \mathrm{C} 4 \mathrm{OH}, \mathrm{C} 5, \mathrm{C} 5: 1, \mathrm{C} 5 \mathrm{DC}, \\
\mathrm{C} 5 \mathrm{OH}, \mathrm{C} 6, \mathrm{C} 8, \mathrm{C} 10, \mathrm{C} 10: 1, \mathrm{C} 10: 2, \mathrm{C} 14, \mathrm{C} 14: 1, \\
\mathrm{C} 16, \mathrm{C} 16 \mathrm{OH}, \mathrm{C} 18, \mathrm{C} 18: 1, \mathrm{C} 18 \mathrm{OH}\end{array}$ \\
\hline Hormones (376) & $\begin{array}{l}\text { Thyroid-Stimulating Hormone, Thyroxine, } \\
\text { Total Galactose, } 17-\alpha \text {-Hydroxyprogesterone }\end{array}$ \\
\hline Immunoreactive Trypsinogen (211) & Immunoreactive Trypsinogen \\
\hline CF DNA (64) & 71 Mutations \\
\hline Hemoglobins $*(73)$ & Varied \\
\hline UDOT $*(65)$ & Varied \\
\hline Galactose-1-phosphate Uridyltransferase (125) & GALT \\
\hline Biotinidase Deficiency (199) & Biotinidase \\
\hline Second Tier CAH (17) & $\begin{array}{l}\text { 17-Hydroxyprogesterone, 4-Androstenedione, } \\
\text { Cortisol, 11-Deoxycortisol, 21-Deoxycortisol }\end{array}$ \\
\hline Lysosomal Storage Disorders * (7) & Pompe, Krabbe \\
\hline SCID (40) & T-cell Excision Circles (TREC) \\
\hline Toxoplasmosis gondii (10) & Anti-Toxoplasma gondii Immunoglobulins $\mathrm{M}$ and $\mathrm{G}$ \\
\hline HIV (28) & Anti-HIV-1 Antibodies \\
\hline
\end{tabular}

* Limited enrollment. 
NSQAP summarizes annual false-positive and false-negative rates for PT challenges for laboratories based on qualitative results (clinical assessments) for analytes or disorders. When false-negative misclassifications are detected, laboratories receive immediate notification from NSQAP supervisory staff so that the source of the error can be investigated and steps can be taken to eliminate the risk of the error occurring again. False-positive results are identified as a tool for use by the laboratory in examining their performance metrics.

\section{NSQAP Quality Control Program}

The NSQAP QC materials allow participants to monitor the long-term stability of their commercial assay kits or in-house methods. They are intended to supplement the participants' method- or kit-control materials. The NSQAP provides NBS laboratories with QC DBS specimens two times per year (Table 4). Sets of DBS QC materials consist of three or four levels of single or multiple analytes. The materials are prepared from pooled, adult donor blood that has been hematocrit-adjusted. Each pool is divided into equal portions so that the sets of QC materials are prepared from the same blood matrix. The whole blood is stored at $-20{ }^{\circ} \mathrm{C}$ for two weeks to lyse the red blood cells. Before dispensing, the blood is thoroughly mixed to assure a homogenous preparation. A customized robotic liquid handling system applies $100-\mu \mathrm{L}$ of whole blood aliquots onto blood collection devices (filter paper). The DBS are dried at ambient temperature overnight and packed the next day by layering each filter paper card between sheets of glassine paper. The QC materials are placed in low-gas permeable bags with several desiccant packets and stored at $-20{ }^{\circ} \mathrm{C}$ until distribution to participating laboratories. The concentration of analyte(s) in each set of QC materials is certified by NSQAP and BMSL and certification pages accompany each shipment of DBS QC materials.

Table 4. NSQAP Quality Control (QC) Programs as of December 2014.

\begin{tabular}{cc}
\hline QC Program (Number of Participants) & Analytes Included in Each Program \\
\hline Amino Acids (398) & Arginine, Leucine, Phenylalanine, Tyrosine, Valine, \\
& Citrulline, Succinylacetone, Methionine, Total Galactose \\
Acylcarnitines (284) & C6, C2, C3, C3DC, C4, C4OH, C5, C5DC, C5OH, \\
& Thyroid-Stimulating Hormone \\
Thyroid-Stimulating Hormone (323) & Thyroxine \\
Thyroxine (77) & 17-Hydroxyprogesterone \\
17-Hydroxyprogesterone (211) & Immunoreactive Trypsinogen \\
Immunoreactive Trypsinogen (185) & GALT \\
Galactose-1-phosphate Uridyltransferase (88) & Pompe, Krabbe, Niemann Pick A/B, Fabry, \\
Lysosomal Storage Disorders (42) & Gaucher, Mucopolysaccharidosis I \\
HIV-1 (28) & Anti-HIV-1 Antibodies \\
X-ALD (4) & $24: 0$ Lysophosphatidylcholine, \\
& $26: 0$ Lysophosphatidylcholine \\
\hline
\end{tabular}




\section{Program Expansions: 2009-2014}

In 2011, the Biochemical Mass Spectrometry Laboratory (BMSL) and the Molecular Quality Improvement Program (MQIP) were established at CDC to support the increasing needs of participants in the fields of mass spectrometry and molecular biology. The BMSL and MQIP collaborate and support the mission of NSQAP to provide quality assurance to newborn screening laboratories.

\subsection{Molecular Quality Improvement Program (MQIP)}

NBS laboratory methods have traditionally involved biochemical assays that detect the presence or absence of analytes or enzyme activities that indicate a particular disorder or subset of disorders. With the introduction of CF mutation screening and more recently SCID screening, the first primary NBS molecular assay, molecular technology has become part of routine NBS. The MQIP collaborates with NSQAP to provide molecular expertise in support of DNA-based PT programs and QA, and works closely with NBS laboratories to assist with the incorporation of primary and second-tier molecular assays into their routine workflow by providing technical assistance and molecular specific resources. The MQIP laboratories use cutting-edge technology to comprehensively characterize disease causing mutations from DBS materials. In addition, MQIP offers a variety of resources for QA to support laboratories using molecular methods for NBS.

The Molecular Assessment Program is an invited personalized site visit to assess the molecular components of the newborn screening laboratory covering all phases of testing including pre-analytical, analytical and post-analytical. The assessment also offers customized approaches for implementing new molecular methods. The MAP team is comprised of scientists from the MQIP, US newborn screening public health programs and a representative from APHL. The NBS Molecular Resources Website is another resource the MQIP has developed in collaboration with APHL [13]. The website contains molecular information tailored to the newborn screening laboratory's needs and includes the MAP site visit portal and checklists, detailed information on molecular assays currently being utilized in newborn screening laboratories in the U.S., a section listing automation resources for molecular screening, and archived materials from past molecular training workshops and webinars. The MQIP also hosts an annual Newborn Screening Molecular Training Course and Laboratory Workshop which consists of both lecture and laboratory segments that directly relate to the detection of newborn disorders using molecular methods.

\subsection{Biochemical Mass Spectrometry Laboratory (BMSL)}

MS/MS has become the standard for the screening of fatty acid, amino acid and organic acid metabolism disorders in newborns worldwide. Largely, laboratories follow the analytical methods described by Chace [7], who introduced the use of MS/MS for the detection of phenylalanine (biomarker for phenylketonuria) in DBS. These methods are used to detect more than fifty diagnostic metabolites, covering over forty metabolic disorders in newborns. Many of these disorders are included in the US Recommended Uniform Screening Panel (RUSP). MS/MS also enables the introduction of second-tier tests to improve disease detection specificity. 
The BMSL, together with the NSQAP and the APHL, provides MS/MS QA services to ensure the quality of MS/MS-based screening activities worldwide. BMSL provides QC and PT DBS materials for galactosemia, amino acids disorders, fatty acid oxidation disorders, organic acid disorders, galactose-1-phosphate uridyltransferase, biotinidase, and second-tier assays for CAH and MSUD. The BMSL consults with public health laboratories to ascertain which markers should be included in NSQAP QA panels. It also houses the filter paper evaluation program, which includes the routine evaluations offered to filter paper manufacturers.

The BMSL and the NSQAP expanded their coverage of MS/MS-detected analytes in the offered QC and PT panels to now cover all primary biomarkers for all MS/MS-detectable core and secondary target disorders listed in the US RUSP. Furthermore, MS/MS-based second-tier testing materials have been developed successfully to improve newborn screening assay specificity for selected biomarkers. These activities, in addition to ensuring the quality of filter paper blood collection devices, support newborn screening laboratory efforts to identify newborns with inborn errors of metabolism, and provide a high level of confidence in the laboratory analytical phase.

\subsection{Training Courses}

One of the most important activities conducted by the CDC is hands-on laboratory trainings, and they are an integral part of the program's mission. To date, our programs have developed three courses that offer an in-depth, hands-on training for NBS laboratorians in order to meet this critical need.

The BMSL offers an in-depth laboratory workshop on newborn screening using MS/MS technology. The 5-day intensive workshop, titled "Newborn Screening by Tandem Mass spectrometry (MS/MS): A Hands-On Course in Understanding Laboratory Issues and Interpreting Test Results.”, is co-sponsored by APHL, and is conducted at the BMSL's laboratories. The course features four MS/MS hands-on exercises designed to enhance understanding of the didactic portions of the workshop. The exercises cover routine MS/MS newborn screening tests, methods and MS source optimization strategies, as well as second-tier testing using MS/MS methods. To date, over 25 laboratorians have benefited from the MS/MS training course.

The MQIP offers a comprehensive hands-on training workshop using molecular technology for newborn screening. This 5-day workshop, titled "The Newborn Screening Molecular Training Workshop" is co-sponsored by APHL and incorporates lecture, group discussion and laboratory activities. The course offers lectures on newborn disorders that include a molecular component such as galactosemia, hemoglobinopathies, CF, MSUD, SCID and MCAD, as well as how to design a molecular laboratory such as setting up unidirectional workflow, comparing genotyping instrument platforms, and molecular data reporting and clinical interpretation. The laboratory activities include DNA extraction, gel electrophoresis, DNA quantitation, PCR-based mutation detection and genotyping, and liquid handling robotics. Since its inception, 52 participants have attended the Molecular Training Workshop.

The NSTRI conducts specialized SCID training for newborn screening programs teaching laboratorians how to produce and analyze DBS reference materials for the TREC assay used to detect SCID. These hands-on workshops are vital for the transfer of technology to states so that they can produce their own reference materials. As of December 2014, over 40 laboratorians have participated in the SCID training workshop. 


\section{Future Directions}

The NSQAP is in constant consultation with its participants and other stakeholders to stay abreast of near and future quality assurance needs. Every candidate analyte is evaluated for stability in the DBS matrix prior to its formal introduction into the NSQAP routine offerings. Depending on the analyte, NSQAP develops analytical methods, or uses commercially available kits to characterize new QA DBS materials. NSQAP sends pilot QA DBS materials to domestic and international partner laboratories that test them with their methods to assure that the materials are suitable for use by newborn screening methods worldwide.

In 2015, BMSL and NSQAP will launch a QA program for X-linked adrenoleukodystrophy (X-ALD), the most common human peroxisomal disorder. Individuals affected with X-ALD exhibit an accumulation of very long-chain fatty acids (VLCFA) due to their reduced oxidation within peroxisomes. The accumulation of C26:0 VLCFA has been shown to result in elevations of C26:0-lysophosphatidylcholine (C26:0-LPC) in X-ALD patients [14]. Currently, New York State is screening its population for X-ALD using the DBS matrix, and other jurisdictions are expected to initiate X-ALD newborn screening in the next two years.

The BMSL and NSQAP will also introduce a new PT program for glucose-6-phosphate dehydrogenase (G6PD) deficiency, the most common human enzyme defect [15]. It affects over 400 million people worldwide, most commonly males due to X-linked heredity. Individuals affected with G6PD deficiency have an increased risk of significant neonatal hyperbilirubinemia due to hemolytic crisis or baseline increase in red blood cell breakdown [16]. It is expected that the G6PD PT program will launch in 2015.

The BMSL is investigating the addition of the amino acids ornithine and glycine for introduction into the NSQAP amino acids QC program. Ornithine is used by one newborn screening program to screen for argininemia through the use of an arginine/ornithine ratio [17]. Glycine levels can be measured by MS/MS in the DBS sample to screen for non-ketotic hyperglycinemia (NKHG). While NKHG is not one of the disorders in the US RUSP, early diagnosis of NKHG might be particularly significant for atypical forms of the disease where early intervention may prove beneficial. Glycine is also measured by some newborn screening laboratories to assist in the diagnosis of NKHG, even though elevated glycine levels may not detectable by MS/MS newborn screening assays [18]. Both amino acids are currently under evaluation for stability and suitability for routine use in the NSQAP amino acids QC materials.

The MQIP is working towards developing a sustainable resource of QA materials to support NSQAP's participants engaged in second-tier mutation detection for specific disorders such as CF and galactosemia. In order to offer molecular QA materials, donor samples that naturally contain the human genome with disease-specific mutations must be collected to test the accuracy of newborn disorder genotyping methods. Thus, QA materials cannot be created without collecting blood from affected donor participants; however, donors are often difficult to find due to the rarity of the mutations. In addition, blood collections only result in a finite number of DBS for QA. Using immortalized cell lines from these rare donors would allow for an infinite supply of a rare resource that can then be used to create DBS that will meet this need. The MQIP in support of the NSQAP is working towards creating DBS made from immortalized cell lines that will work robustly with different DNA extraction methods and a wide variety of genotyping methods and platforms. Once validated, it will be feasible for NSQAP to provide PT and PT and QC materials for screening methods that involve genotyping a panel of mutations. 


\section{Discussion}

NSQAP is a proactive, multi-component QA program for a specialized area of public health testing [2]. NSQAP, BMSL, MQIP and NSTRI provide PT testing, QC materials, filter paper evaluations, special consultations, and technical assistance to public health and private laboratories engaged in NBS. The PT data-reporting is handled by an internet web site that permits participants to report their results online for each analyte, receive timely performance evaluation reports, and allows storage and retrieval access for historical data-report summaries . The NSQAP serves as a focal point for NBS laboratories to address a variety of problems that may be encountered with the performance of laboratory-developed tests, and commercial kits/products with their manufacturers. Prompt consultation with the laboratories participating in the NSQAP may reduce the risk of catastrophic consequences of a delayed diagnosis to the newborn.

The NSQAP, BMSL, MQIP and NSTRI are designed to help screening laboratories achieve excellent technical proficiency and maintain confidence in their performance while processing large volumes of specimens daily. The programs continually strive to produce certified DBS materials for reference and QC analysis, to improve the quality and scope of services, and to provide immediate consultative and technical assistance. Over 14 million DBS QA materials have been distributed globally during 36 years of operation. Through our interactive efforts with the program's participants, it works to meet their growing and changing NBS needs, always with an eye on future technological advances. The accuracy of screening tests marks the difference between life and death for many babies; in other instances, identifying newborns with a disorder means that they can be treated and thus avoid life-long disability or severe cognitive impairment. In the United States, over 12,000 babies each year are saved from deleterious health outcomes through newborn screening and early identification. Thousands of newborns and their immediate families have benefited from reliable and accurate testing that has been accomplished by a network of screening laboratories and the NSQAP, BMSL, MQIP and NSTRI.

\section{Acknowledgments}

The authors would like to thank the staff of the Newborn Screening and Molecular Biology Branch at the US Centers for Disease Control and Prevention. The opinions expressed by authors contributing to this journal do not necessarily reflect the opinions of the Centers for Disease Control and Prevention, or the authors' affiliated institutions. Use of trade names is for identification only and does not imply endorsement.

\section{Author Contributions}

V.D.J. designed the overall concept, and contributed the majority of the writing of the manuscript. J.V.M, S.K.C. and C.D.C. contributed writing and editing of the manuscript.

\section{Conflicts of Interest}

The authors declare no conflict of interest. 


\section{References}

1. US Centers for Disease Control and Prevention. Ten great public health achievements--United States, 2001-2010. MMWR 2011, 60, 619-623.

2. De Jesus, V.R.; Mei, J.V.; Bell, C.J.; Hannon, W.H. Improving and assuring newborn screening laboratory quality worldwide: 30-year experience at the Centers for Disease Control and Prevention. Semin. Perinatol. 2010, 34, 125-133.

3. Zobel, S. Newborn Screening Quality Assurance Program 2012 Annual Summary Report. Zobel, S., Ed. US Centers for Disease Control and Prevention: Atlanta, GA, USA, 2013; Volume 30.

4. Clinical Laboratory Improvement Amendments (CLIA). Available online: http://www.cms.gov/Regulations-and-Guidance/Legislation/CLIA/index.html (accessed on 13 April 2015).

5. Clinical and laboratory Standards Institute (CLSI). NBS01-A6 Blood Collection on Filter Paper for Newborn Screening Programs; Approved Standard - Sixth Edition; CLSI: Wayne, PA, USA, 2013.

6. Gwinn, M.; Pappaioanou, M.; George, J.R.; Hannon, W.H.; Wasser, S.C.; Redus, M.A.; Hoff, R.; Grady, G.F.; Willoughby, A.; Novello, A.C.; et al. Prevalence of HIV infection in childbearing women in the United States. Surveillance using newborn blood samples. JAMA 1991, 265, 1704-1708.

7. Chace, D.H.; Millington, D.S.; Terada, N.; Kahler, S.G.; Roe, C.R.; Hofman, L.F. Rapid diagnosis of phenylketonuria by quantitative analysis for phenylalanine and tyrosine in neonatal blood spots by tandem mass spectrometry. Clin. Chem. 1993, 39, 66-71.

8. Mei, J.V.; Li, L.; Rasmussen, S.A.; Collier, S.; Frias, J.L.; Honein, M.A.; Shawe, G.M.; Lorey, F.; Meyerg, R.; Chaing, S.; et al. Effect of specimen storage conditions on newborn dried blood spots used to assess Toxoplasma gondii immunoglobulin M (IgM). Clin. Chim. Acta 2011, 412, 455-459.

9. Lacey, J.M.; Minutti, C.Z.; Magera, M.J.; Tauscher, A.L.; Casetta, B.; McCann, M.; Lymp, J.; Hahn, S.H.; Rinaldo, P.; Matern, D. Improved specificity of newborn screening for congenital adrenal hyperplasia by second-tier steroid profiling using tandem mass spectrometry. Clin. Chem. 2004, 50, 621-625.

10. De Jesus, V.R.; Zhang, X.K.; Keutzer, J.; Bodamer, O.A.; Muhl, A.; Orsini, J.J.; Caggana, M.; Vogt, R.F.; Hannon, W.H. Development and evaluation of quality control dried blood spot materials in newborn screening for lysosomal storage disorders. Clin. Chem. 2009, 55, 158-164.

11. Adam, B.W.; Flores, S.R.; Hou, Y.; Allen, T.W.; de Jesus, V.R. Galactose-1-Phosphate uridyltransferase dried blood spot quality control materials for newborn screening tests. Clin. Biochem. 2015, doi:10.1016/j.clinbiochem.2014.12.009.

12. US Department of Health and Human Services Secretary's Advisory Committee on Heritable Disorders in Newborns and Children. Recommended Uniform Screening Panel. Available online: http://www.hrsa.gov/advisorycommittees/mchbadvisory/heritabledisorders/recommendedpanel/in dex.html (accessed on 22 December 2014).

13. Association of Public Health Laboratories. Molecular Resources. Available online: http://www.aphl.org/aphlprograms/newborn-screening-and-genetics/molecular/pages/default.aspx (accessed on 21 January 2015). 
14. Hubbard, W.C.; Moser, A.B.; Liu, A.C.; Jones, R.O.; Steinberg, S.J.; Lorey, F.; Panny, S.R.; Vogt, R.F.; Macaya, D.; Turgeon, C.T.; et al. Newborn screening for X-linked adrenoleukodystrophy (X-ALD): validation of a combined liquid chromatography-tandem mass spectrometric (LC-MS/MS) method. Mol. Gen. Metabol. 2009, 97, 212-220.

15. Bernardo, J.; Nock, M. Pediatric Provider Insight into Newborn Screening for Glucose-6-Phosphate Dehydrogenase Deficiency. Clin. Peds. 2014, doi:10.1177/0009922814557786.

16. Beutler, E. Glucose-6-Phosphate dehydrogenase deficiency: A historical perspective. Blood 2008, $111,16-24$.

17. Jay, A.; Seeterlin, M.; Stanley, E.; Grier, R. Case Report of Argininemia: The Utility of the Arginine/Ornithine Ratio for Newborn Screening (NBS). JIMD Rep. 2013, 9, 121-124.

18. Tan, E.S.; Wiley, V.; Carpenter, K.; Wilcken, B. Non-Ketotic hyperglycinemia is usually not detectable by tandem mass spectrometry newborn screening. Mol. Gen. Metabol. 2007, 90, 446-448.

(C) 2015 by the authors; licensee MDPI, Basel, Switzerland. This article is an open access article distributed under the terms and conditions of the Creative Commons Attribution license (http://creativecommons.org/licenses/by/4.0/). 